Received: 2015.08 .21 Accepted: 2015.10.22 Published: 2016.02 .11

\title{
Deceased Donor Kidney Transplantation in the Eurotransplant Senior Program (ESP): A Single-Center Experience from 2008 to 2013
}

Authors' Contribution Study Design A Data Collection B Statistical Analysis C Data Interpretation D Manuscript Preparation E Literature Search F Funds Collection $\mathrm{C}$
ABCDEFG 1 Johannes Jacobi

BCF 1 Sophie Beckmann

DF 1 Katharina Heller

CDE 1 Karl F. Hilgers

D 2 Hendrik Apel

BD 3 Bernd Spriewald

DE 1 Kai-Uwe Eckardt

BCDE 4 Kerstin U. Amann
1 Department of Nephrology and Hypertension, Friedrich-Alexander-University Erlangen-Nürnberg, Erlangen, Germany

2 Department of Urology, Friedrich-Alexander-University Erlangen-Nürnberg, Erlangen, Germany

3 Department of Hematology and Oncology, Friedrich-Alexander-University Erlangen-Nürnberg, Erlangen, Germany

4 Department of Nephropathology, Friedrich-Alexander-University Erlangen-Nürnberg, Erlangen, Germany
Corresponding Author: Source of support:
Johannes Jacobi, e-mail: johannes.jacobi@uk-erlangen.de

Departmental sources

Background: The aim of this study was to evaluate the outcome after transplantation of deceased allografts in donor/recipient pairs aged $\geq 65$ years enrolled in the Eurotransplant Senior Program (ESP).

Material/Methods: In this retrospective cohort study we evaluated data from 89 patients transplanted under the ESP protocol from 2008 to 2013. Outcome parameters included graft and patient survival, rate of biopsy-proven acute rejections (BPAR), peri- and post-operative complications, tumor development, development of donor-specific antibodies (DSA), and the prognostic role of preimplantation biopsies.

Results: $\quad$ One-year patient and allograft survival rates were $92.1 \%$ and $84.3 \%$, respectively. During follow-up, 23 (26\%) patients died; the major cause of death was sepsis, followed by cardiovascular events and malignancies. BPAR episodes were frequent within the first year $(\sim 33 \%)$ and overall were less common in patients treated with tacrolimus. Post-transplant malignancies were seen in 15 (17\%) patients. During follow-up, 16 (18\%) patients developed DSA; patients with delayed graft function (DGF) were more likely to develop DSA $(p=0.029)$. A higher preimplantation biopsy score was associated with DGF but did not predict later graft outcome.

Conclusions: The results highlight increased risks in ESP transplant candidates and the importance of careful surveillance of this patient group.

MeSH Keywords: Graft Rejection • Kidney Transplantation • Survival Rate

Full-text PDF: http://www.annalsoftransplantation.com/abstract/index/idArt/895731

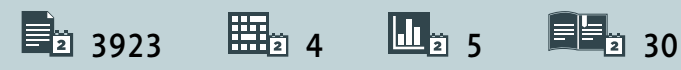




\section{Background}

Increased aging of the dialysis population has made it challenging to identify patients that are suitable candidates for kidney transplantation. The main challenge remains to carefully select individuals in which the benefits of solid organ transplantation outweigh potential harms and risks such as deterioration of underlying comorbidities or adverse events associated with immunosuppression in elderly patients.

Thus, despite all efforts, mortality in transplant recipients aged 65 years and older remains high, with annual death rates of 20\% [1]. Not surprisingly, only 2 decades ago less than 15\% of all deceased renal allografts were transplanted into recipients age $\geq 65$ years [2].

Nonetheless, comparing the outcome of aged transplant recipients with that of patients remaining on dialysis undoubtedly shows improved survival rates for patients being transplanted [2-4]. In the landmark study by Wolfe et al., life expectancy doubled in transplant recipients age $>65$ years compared to those that remained on the waiting list; the gain in lifetime exceeded 4 years [2].

In 1999 the Eurotransplant Senior Program (ESP) was launched within the Eurotransplant kidney allocation scheme. The program aimed to allow efficient use of kidneys from older donors and to offer elderly patients access to transplantation, whereby local allocation of organs was used to keep cold-ischemic time low [5]. Encouraging results come from a 5 -year analysis of the ESP including 1404 patients [6]. The number of elderly donors dramatically increased and cold ischemic time was reduced by $\sim 5$ hours. More importantly, despite slightly higher rejection rates, graft and patient survival were similar in ESP patients and those allocated within the regular program [6]. Given these findings, an age limitation for organ donors no longer appears to be justified. Nevertheless, $60 \%$ of kidneys retrieved from deceased organ donors aged $>65$ years are still being discarded in the United States [7].

In the present retrospective, single-center, cohort study we meticulously analyzed a variety of outcome parameters such as patient and graft survival rates, rejection episodes, peri- and post-operative complications, risk of infection and tumor development under immunosuppression, development of donorspecific antibodies following transplantation, and the prognostic value of preimplantation biopsies of renal allografts in patients enrolled into our ESP program over a 5-year period from 2008 to 2013.

\section{Material and Methods}

\section{Study cohort}

In this single-center, retrospective, cohort study all patients $\geq 65$ years that received their first renal allograft from a deceased donor $\geq 65$ years between 2008 and 2013 were included. Patients who received dual kidney transplantation $(n=15)$ were excluded. Patients were referred from $\sim 40$ different nonprofit or for-profit dialysis centers, as described earlier [8]. All deceased donations were donations after brain death from heart-beating donors.

Checking for scenarios of aged recipients ( $\geq 65$ years) receiving allografts from donors aged $<65$ years $(n=7)$ and patients $<65$ years receiving kidneys from donors $\geq 65$ years $(n=7)$ yielded too few patients to allow meaningful comparison with the ESP group. Thus, our analysis is solely based on the latter group of patients.

Baseline triple immunosuppression included a calcineurin inhibitor (CNI, either tacrolimus or cyclosporine), antimetabolite (mycophenolate-sodium or mycophenolate mofetil), and steroids, as previously described [8]. Baseline $\mathrm{CNI}$ and induction therapy were either predetermined in patients enrolled into an interventional study protocol or based on the underlying immunological risk, as well as patient metabolic profile [8]. Target trough levels at 6 and 12 months after transplantation for cyclosporine and tacrolimus were $50-80 \mathrm{ng} / \mathrm{ml}$ and $40-70 \mathrm{ng} / \mathrm{ml}$, and $4-6 \mathrm{ng} / \mathrm{ml}$ and $3-5 \mathrm{ng} / \mathrm{ml}$, respectively. Early steroid withdrawal within 2 weeks after transplantation was only performed in patients enrolled into a clinical trial; otherwise, steroids were tapered to reach a dose of 2.5 $\mathrm{mg} /$ day after 6 months.

Baseline characteristics of transplant recipients and transplantrelevant data were saved in a transplant database using SPSS (version 21.0). All patients gave their written informed consent for data collection and analysis prior to transplantation. All data were collected in strictly pseudonymous form. Based on the retrospective nature of this cohort study, we did not need ethics approval from our local ethics committee.

\section{Patient follow-up}

During the first year most patients were closely followed in our outpatient clinic. CMV prophylaxis, as well as PCR screening for CMV or BK (polyoma) virus, was performed as recommended by KDIGO guidelines [9]. Thereafter, patients were seen in the transplant center at least on a yearly basis, at which time-point screening for HLA alloantibodies (ELISA) was performed in all patients. Confirmatory testing for samples testing positive for 
HLA class I and/or II antibodies was performed using the fluorescent bead method (Luminex).

During follow-up, peri- and post-operative complications, as well as infections, tumor development, and biopsy-proven acute rejections (BPAR), were recorded.

Missing data of patients that did not come to their routine visit in our clinic were retrieved by contacting the local treating physician.

\section{Histological analysis of preimplantation biopsies}

Preimplantation (zero-hour) biopsies were performed during back-table preparation of the kidneys, as described earlier [8]. The histological findings were thus not available prior to transplantation and therefore did not aid the decision process. Preimplantation, as well as all other biopsies obtained during follow-up, were analyzed and graded according to BANFF 2009 classification by 2 experienced nephropathologists, as described earlier [8]. In addition, the degree of tubular atrophy, interstitial fibrosis, glomerulosclerosis, and arterio-arteriolar hyalinosis was classified separately as absent (0), mild (1), moderate (2) or severe (3) [8]. In each patient the sum of these 4 scores was calculated; thus, the maximum score was 12. In sub-analyses, patients with a score $<3$ were compared to those with a score of 3-5 or $>5$.

\section{Statistical analysis}

Continuous variables were summarized using descriptive statistics. Categorical variables were summarized using frequency tables and analyzed using $\chi^{2}$ test. All data are expressed as mean \pm standard deviation (SD). The paired t-test was used to analyze differences within treatment groups. Patient and allograft survival curves, as well as BPAR rates, were computed according to the method of Kaplan-Meier. Multivariate linear regression analysis was performed to determine independent factors influencing allograft function (eGFR at 1 year) and BPAR. Values above bar graphs represent the variable mean. Statistical significance was accepted at a value of $p<0.05$ (2-sided)

\section{Results}

\section{Study cohort}

Baseline characteristics of organ donors and transplant recipients, as well as transplant-relevant data, are shown in Table 1. A total of 89 patients were included in the final analysis. Of these, 68 were male and 21 female. Mean dialysis time was 43 months, and mean time on a waiting list was 28 months.
Table 1. Baseline characteristics of organ donors and transplant recipients and transplant relevant data.

\begin{tabular}{|c|c|}
\hline Variable & Patients $(n=89)$ \\
\hline Recipient age (years) & $68.2 \pm 3.2$ \\
\hline Recipient sex (m/f) & $68 / 21$ \\
\hline Recipient BMI $\left(\mathrm{kg} / \mathrm{m}^{2}\right)$ & $26.3 \pm 3.6$ \\
\hline Waitlist time (months) & $28 \pm 25$ \\
\hline Dialysis vintage (months) & $43 \pm 26$ \\
\hline RRT (HD/CAPD/preemptive) (n) & $83 / 5 / 1$ \\
\hline Donor age (years) & $71.5 \pm 4.6$ \\
\hline Donor sex $(m / f)$ & $42 / 47$ \\
\hline Donor BMI (kg/m²) & $27.4 \pm 5.2$ \\
\hline $\begin{array}{l}\text { Donor creatinine on admission to hospital } \\
(\mathrm{mg} / \mathrm{dl})\end{array}$ & $0.88 \pm 0.30$ \\
\hline $\begin{array}{l}\text { Donor creatinine prior to organ } \\
\text { procurement }(\mathrm{mg} / \mathrm{dl})\end{array}$ & $1.00 \pm 0.50$ \\
\hline $\begin{array}{l}\text { Donor eGFR prior to organ procurement } \\
\text { (ml/min) }\end{array}$ & $77.7 \pm 32.5$ \\
\hline Cold ischemic time (hours) & $11.0 \pm 3.7$ \\
\hline Warm ischemic time (min) & $45 \pm 19$ \\
\hline HLA-mismatch (n) & $3.9 \pm 1.3$ \\
\hline Use of tacrolimus as initial CNI (n /\%) & $53 / 59.6 \%$ \\
\hline Use of cyclosporine A as initial CNI (n /\%) & $36 / 40.4 \%$ \\
\hline ATG-induction (n /\%) & $17 / 19.1 \%$ \\
\hline IL2-induction (n /\%) & $72 / 80.9 \%$ \\
\hline $\begin{array}{l}\text { Steroid-free immunosuppression after } 1 \\
\text { week }(n / \%)\end{array}$ & $11 / 12.4 \%$ \\
\hline PRAs (n/\%) & $3 / 3.4 \%$ \\
\hline Study participant (n /\%) & $28 / 31.5 \%$ \\
\hline Primary function (n /\%) & $56 / 62.9 \%$ \\
\hline Delayed graft function ( $\mathrm{n} / \%$ ) & $24 / 27.0 \%$ \\
\hline $\begin{array}{l}\text { Permanent non-function, early graft loss or } \\
\text { death }(n / \%)\end{array}$ & $9 / 10.1 \%$ \\
\hline $\begin{array}{l}\text { Creatinine at discharge from hospital } \\
(\mathrm{mg} / \mathrm{dl})\end{array}$ & $2.5 \pm 1.2$ \\
\hline eGFR at discharge from hospital ( $\mathrm{ml} / \mathrm{min})$ & $28.1 \pm 11.3$ \\
\hline Hospitalization time (days) & $21.7 \pm 16.8$ \\
\hline
\end{tabular}

Major categories of underlying renal disease were: unknown ( $26 \%)$, biopsy-proven glomerulonephritis ( 21\%), hypertensive nephropathy ( 16\%), autosomal polycystic kidney disease 
Table 2. Time-point and cause of death in ESP transplant recipients.

\begin{tabular}{|c|c|c|c|}
\hline Age at $T x / \operatorname{sex}$ & $\begin{array}{l}\text { Organ function } \\
\text { at time of death }\end{array}$ & $\begin{array}{l}\text { Time-point of death } \\
\text { (days after Tx) }\end{array}$ & Cause of death \\
\hline $69 m$ & Yes & 1 & Pulmonary embolism \\
\hline $65 \mathrm{~m}$ & Yes & 53 & Suicide \\
\hline $74 f$ & No & 74 & Pulmonary embolism \\
\hline $66 f$ & No & 89 & Myocardial infarction \\
\hline $68 m$ & Yes & 210 & Pneumonia/sepsis \\
\hline $65 \mathrm{~m}$ & Yes & 330 & Myocardial infarction \\
\hline $69 m$ & No & 366 & Stroke \\
\hline $65 f$ & No & 388 & Meningitis/sepsis \\
\hline $70 m$ & No & 440 & Osteomyelitis/sepsis \\
\hline $67 m$ & Yes & 469 & Pancreatic cancer \\
\hline $65 \mathrm{~m}$ & Yes & 490 & Stroke \\
\hline $77 m$ & Yes & 555 & CMV-encephalitis \\
\hline $67 \mathrm{~m}$ & No & 581 & Esophageal cancer \\
\hline $66 f$ & No & 601 & Unknown \\
\hline $66 f$ & Yes & 700 & Pneumocystis pneumonia \\
\hline $65 \mathrm{~m}$ & No & 1143 & Septic shock \\
\hline $70 f$ & No & 1213 & Septic schock \\
\hline $69 m$ & No & 1229 & Esophageal cancer \\
\hline $68 m$ & Yes & 1251 & PTLD (EBV-associated) \\
\hline $67 m$ & Yes & 1379 & Pneumonia/sepsis \\
\hline $65 \mathrm{~m}$ & Yes & 1808 & Endocarditis/sepsis \\
\hline $65 \mathrm{~m}$ & Yes & 1836 & Pneumonia/sepsis \\
\hline $66 f$ & Yes & 1983 & Pancreatic cancer \\
\hline
\end{tabular}

( 15\%), diabetic nephropathy ( 11\%), and tubulo-interstitial nephritis $(\sim 5)$. Mean follow-up of all patients was $3.9 \pm 1.6$ years and all living patients were followed for at least 12 months after transplantation.

\section{Impact of cold ischemic time}

Because cold ischemic time is known to have a negative impact on graft and patient survival, as well as the risk of BPAR, we separately analyzed kidneys coming from the same deceased donor that were transplanted consecutively into 2 recipients at our transplant center. Transplantations were performed successively for logistic reasons.
A total of 40 kidneys from 20 donors were analyzed and the outcome of the first versus second transplant was compared. Cold ischemic time was significantly lower in patients transplanted first $(7.6 \pm 2.1$ vs. $13.4 \pm 2.9$ hours, $p<0.0001)$. Groups did not differ in terms of baseline immunosuppression or induction therapy. Graft function, rate of DGF, and graft and patient survival were not affected by rank of transplantation (data not shown). No difference in BPAR episodes was observed.

\section{Patient and graft survival}

During follow-up, 23 (26\%) patients died. Patient survival at 1 year was $\sim 92 \%$ (7 deaths within the first year). An additional 16 patients died thereafter; causes and time-point of death after 
A

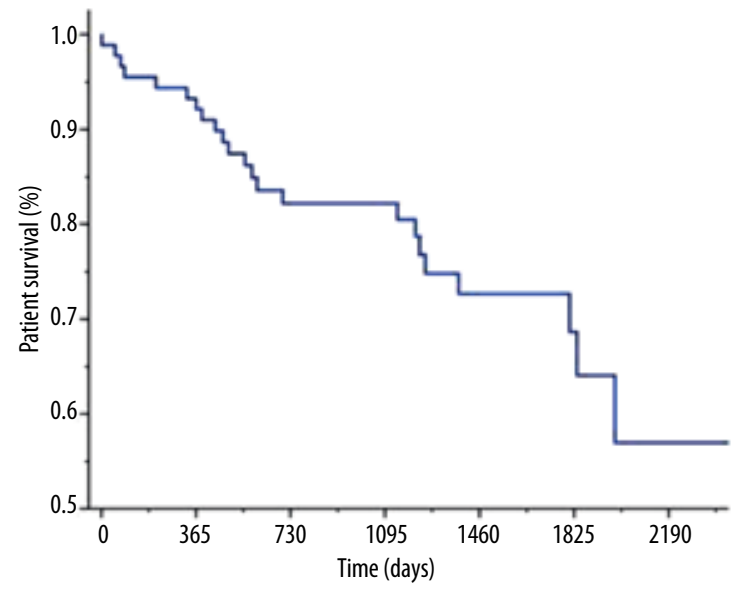

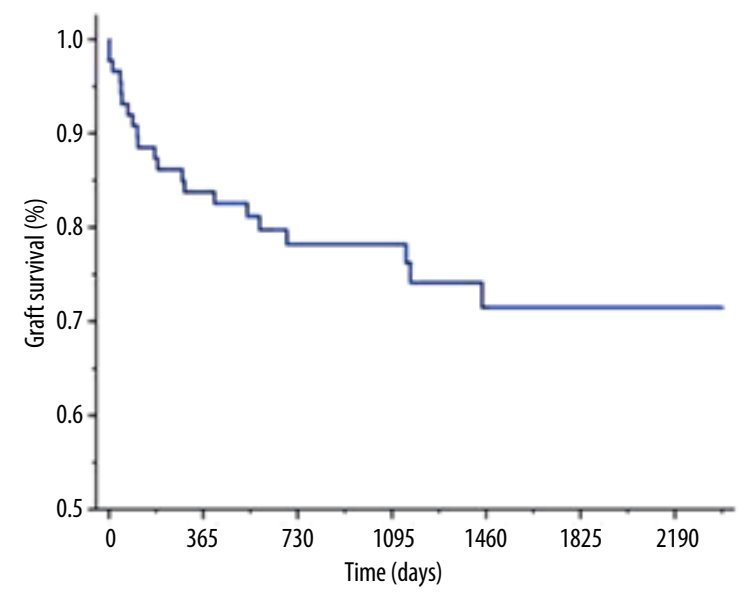

Figure 1. (A) Patient survival over time. (B) Graft survival over time (censored for death with functioning graft).

A

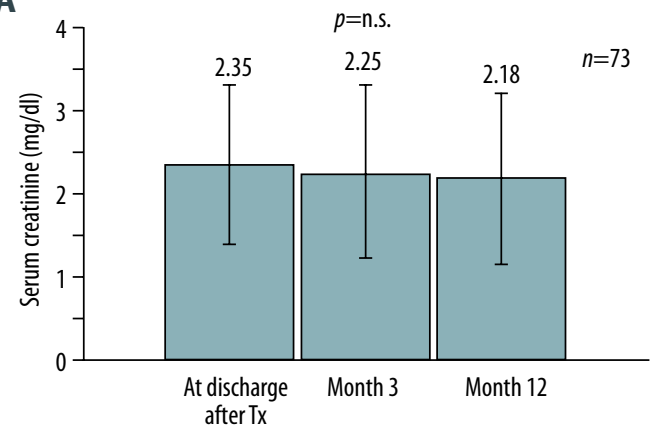

B

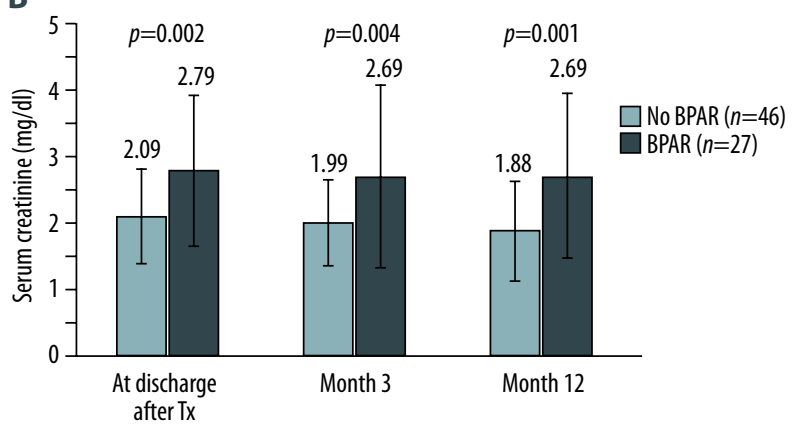

Figure 2. (A) Serum creatinine within the first year after transplantation. (B) BPAR and serum creatinine within the first year after transplantation.

transplantation are depicted in Table 2. Three major causes of death were identified: infections with sepsis-related death (10 cases), cardiovascular events (6 cases), and malignancies (5 cases). A total of 13 patients died with a functioning allograft, whereas the remaining patients died with loss of organ function. The Kaplan-Meier survival curve is shown in Figure $1 \mathrm{~A}$.

Patient survival at 5 years was $\sim 65 \%$. Allograft survival (censored for death with functioning graft) after 1 and 5 years was $\sim 84$ and $\sim 72 \%$, respectively (Figure $1 \mathrm{~B}$ ).

\section{Allograft function over time}

In 73 (82\%) patients, serial creatinine measurements over the first year after transplantation were available. We observed a slight, but non-significant, decrease of serum creatinine over time (Figure 2A). Patients with BPAR episodes within the first year after transplantation had a worse outcome with regard to allograft function (Figure 2B). Notably, donor and recipient ages did not differ between these groups. In patients with complete follow-up for 2-5 years, serum creatinine tended to slightly decrease over time, but remained fairly stable overall (data not shown).

In linear regression analyses, predictors of 1-year eGFR were donor age $(\beta-0.30, T$-value $-2.61, p=0.012)$, and BPAR $(\beta-0.30$, T-value $-2.43, p=0.018)$

\section{Peri- and postoperative complications}

Peri- and post-operative complications (either surgical or nonsurgical) are depicted in Table 3 and were observed in 41 (46\%) patients. Patients with complications had a longer hospitalization compared to those without complications ( $26 \pm 22 \mathrm{vs}$. $18 \pm 8$ days, $p=0.028)$. The 4 most common complications following transplantation were: lymphoceles (20 patients) with need for laparoscopic surgical drainage (11 patients), re-surgery for postoperative bleeding (11 patients), postoperative hernia (11 patients), and development of new-onset diabetes after transplantation (NODAT, 10 patients). Recipients with a 
baseline $\mathrm{BMI}$ of $>30 \mathrm{~kg} / \mathrm{m}^{2}(\mathrm{n}=16)$ were at markedly higher risk for hernia development $(37.5 \%$ vs. $6.8 \%$ in patients with a baseline $\left.\mathrm{BMI}<30 \mathrm{~kg} / \mathrm{m}^{2}, \mathrm{p}=0.004\right)$. Similarly, lymphocele formation was more frequent in obese patients (43.8 vs. $17.8 \%$, $\mathrm{p}=0.032$ ). Notably, donor BMI and baseline CNI were not associated with lymphocele formation.

\section{Post-transplant tumor development}

During follow-up, a diagnosis of tumor disease was made in $15(-17 \%)$ patients: 7 patients with skin tumors (4 basalioma and 3 squamous cell carcinoma), 1 patient with PTLD disease, 2 patients with pancreatic cancer, 2 patients with esophageal cancer, and 1 patient each with renal cell (native kidney) thyroid and colon cancer. The mean time at which the tumor was diagnosed after transplantation was $1.8 \pm 1.3$ years, and the earliest tumor (thyroid cancer) was diagnosed 190 days after transplantation.

\section{CMV and BK viral infection}

During follow-up, CMV viremia of at least $10^{3}$ copies $/ \mathrm{ml}$ was observed in 28 (31.5\%) patients. The risk was lowest in $\mathrm{D}^{-} / \mathrm{R}^{-}$ patients ( 2 out of 9 , or $22 \%$ ), followed by $D^{+} / R^{+}$(10 out of 40 , or $25 \%$ ), $D^{-} / R^{+}$( 6 out of 20 , or $30 \%$ ), and $D^{+} / R^{-}$(10 out of 20 , or $50 \%, \chi^{2} p=$ n.s.).

BK viral replication of at least $10^{3}$ copies $/ \mathrm{ml}$ was observed in $19(21 \%)$ patients. Maximal viral loads of $>10^{3},>10^{4},>10^{5}$, and $>10^{6}$ were seen in $3,9,4$, and 3 patients, respectively. All patients with BK viral replication underwent renal biopsy; a diagnosis of polyomavirus-associated nephropathy (PyVAN) was made in $53 \%$ of all cases with BK viremia. Average duration of BK viral replication was $4.5 \pm 2.5$ months.

\section{HLA alloantibodies and donor-specific antibodies}

Prior to transplantation, only 1 patient had class II HLA antibodies detected by ELISA. During follow-up, 22 (24.7\%) patients developed class I or II HLA-antibodies (ELISA); 17 of these patients had received blood transfusions during followup (Table 4). Overall, 55 (61.8\%) patients received blood transfusions during follow-up.

Nine patients tested positive for both HLA class I and II antibodies. In these subjects, DSA against class I or class II antigens was verified in 8 and 6 subjects, respectively (Table 4). In 3 subjects, renal biopsy revealed histological evidence for acute antibody-mediated rejection (peritubular capillaritis with positive C4d staining).

Out of 5 patients who tested positive only for class I HLA antibodies, class I DSA was documented by Luminex in 1 subject,
Table 3. Peri-postoperative complications in ESP transplant recipients.

\begin{tabular}{|c|c|}
\hline Complication & Patients (n/\%) \\
\hline Lymphocele & $20 / 22.5 \%$ \\
\hline Conservative management & $9 / 45 \%$ \\
\hline Laparoscopic lympocele drainage & $11 / 55 \%$ \\
\hline $\begin{array}{l}\text { Postoperative bleeding with surgical } \\
\text { revision }\end{array}$ & $11 / 12.4 \%$ \\
\hline Hernia & $11 / 12.4 \%$ \\
\hline NODAT & $10 / 11.2 \%$ \\
\hline Ureter neoimplantation & $7 / 7.9 \%$ \\
\hline TURP & $5 / 7.4 \%$ \\
\hline Transplant renal artery stenosis & $4 / 4.5 \%$ \\
\hline Urinoma & $3 / 3.4 \%$ \\
\hline Perioperative myocardial infarction & $3 / 3.4 \%$ \\
\hline Perfusion deficit & $2 / 2.2 \%$ \\
\hline Renal vein thrombosis & $2 / 2.2 \%$ \\
\hline Renal artery dissection & $1 / 1.1 \%$ \\
\hline Perioperative deep vein thrombosis & $1 / 1.1 \%$ \\
\hline Renal artery thrombosis & $1 / 1.1 \%$ \\
\hline Bowel perforation & $1 / 1.1 \%$ \\
\hline
\end{tabular}

and 2 patients showed a putative DSA against HLA Cw locus, for which donor HLA-typing was missing (Table 4).

Out of 8 patients who only tested positive for class II HLA antibodies, class II DSA was documented by Luminex in 6 subjects (Table 4). Antibody-mediated rejection was documented in 2 patients.

Overall, most HLA antibodies were directed against HLA class II antigens, especially against HLA-DQ epitopes. Interestingly, patients with delayed graft function or permanent non-function were more likely to develop DSA during follow-up (Figure 3).

\section{Biopsy-proven acute rejections}

During follow-up, 33 (37\%) patients had BPAR. In almost all cases ( $>90 \%$ ) these rejections occurred within the first year or within the first 6 months after transplantation (see Figure 4). Eighteen patients had mild cellular BANFF IA rejections, and 7 of these patients had more than 1 episode of this type. Three patients had more severe BANFF IB rejection, 12 had mild-tomoderate vascular BANFF IIA, and 2 had BANFF IIB rejections. 
Table 4. Development of HLA class I and II antibodies and DSA in ESP transplant recipients.

\begin{tabular}{|c|c|c|c|c|c|c|c|c|c|c|}
\hline Age/sex & HLA-MM & PRA & $\begin{array}{l}\text { DGF } \\
\text { or } \\
\text { PNF }\end{array}$ & $\begin{array}{l}\text { Time of detection } \\
\text { of HLA antibodies } \\
\text { (days after Tx) }\end{array}$ & $\begin{array}{c}\text { Blood } \\
\text { transfusion } \\
\text { (days after Tx) }\end{array}$ & $\begin{array}{l}\text { HLA } \\
\text { class I }\end{array}$ & $\begin{array}{c}\text { HLA } \\
\text { class II }\end{array}$ & $\begin{array}{l}\text { DSA } \\
\text { class I }\end{array}$ & $\begin{array}{c}\text { DSA } \\
\text { class II }\end{array}$ & AMR \\
\hline $73 m$ & $1-1-0$ & $0 \%$ & + & 149 & $+(160)$ & + & + & A24 & - & - \\
\hline $65 f$ & $1-1-1$ & $0 \%$ & + & 348 & $+(73)$ & + & + & A3 & DR15 & - \\
\hline $71 \mathrm{~m}$ & $1-1-0$ & $0 \%$ & + & $\begin{array}{c}349 \\
\text { loss of allograft } \\
1 \text { day after Tx }\end{array}$ & $+(1)$ & + & + & $\mathrm{A} 1, \mathrm{~A} 32, \mathrm{~B} 44$ & - & $(-)$ \\
\hline $77 \mathrm{~m}$ & $1-2-1$ & $0 \%$ & + & 359 & $+(43)$ & + & + & - & DQ7,DQ8,DQ9 & + \\
\hline $66 f$ & $1-2-2$ & $0 \%$ & - & 436 & $+(246)$ & + & + & $\mathrm{A} 11, \mathrm{~B} 52, \mathrm{~B} 62$ & DR1,DR4,DQ8 & - \\
\hline $67 m$ & $1-1-2$ & $0 \%$ & + & $\begin{array}{c}514 \\
\text { loss of allograft } \\
1 \text { day after Tx }\end{array}$ & $+(1)$ & + & + & B41 & DR15,DQ6 & $(-)$ \\
\hline $70 f$ & $0-1-1$ & $0 \%$ & - & 1176 & $+(1111)$ & + & + & B41,B49,B50 & DQ8,DQ9 & + \\
\hline $67 m$ & $1-2-2$ & $0 \%$ & + & 1249 & $+(14)$ & + & + & B63 & DQ2 & + \\
\hline $68 m$ & $0-2-1$ & $0 \%$ & - & 1599 & - & + & + & B57 & - & - \\
\hline $66 f$ & $1-2-0$ & $0 \%$ & + & 2 & $+(1)$ & + & - & A26, B55 & - & - \\
\hline $69 m$ & $2-1-2$ & $0 \%$ & - & 578 & $+(371)$ & + & - & $-(\mathrm{Cw})^{*}$ & - & - \\
\hline $65 \mathrm{~m}$ & $2-2-1$ & $0 \%$ & - & 1409 & $+(84)$ & + & - & $-(C w)^{*}$ & - & - \\
\hline $65 \mathrm{~m}$ & $1-2-1$ & $4 \%$ & + & 1624 & $+(825)$ & + & - & - & - & - \\
\hline $65 f$ & $2-0-2$ & $0 \%$ & - & 2112 & - & + & - & - & - & - \\
\hline $70 m$ & $1-2-2$ & $0 \%$ & - & 155 & $+(123)$ & - & + & - & $\mathrm{DQ} 2$ & + \\
\hline $65 \mathrm{~m}$ & $1-2-1$ & $0 \%$ & + & 397 & $+(1)$ & - & + & - & DQ7,DQ8,DQ9 & - \\
\hline $66 \mathrm{~m}$ & $2-2-1$ & $0 \%$ & - & 453 & - & - & + & - & DQ5 & - \\
\hline $66 f$ & $1-2-1$ & $70 \%$ & + & 508 & $+(3)$ & - & + & - & DQ6 & + \\
\hline $72 m$ & $0-2-1$ & $0 \%$ & - & 839 & $+(1)$ & - & + & - & DQ1 & - \\
\hline $65 f$ & $0-2-1$ & $0 \%$ & - & 947 & - & - & + & - & - & - \\
\hline $72 m$ & $0-1-1$ & $0 \%$ & + & 1008 & - & - & + & - & DQ3 & - \\
\hline $71 \mathrm{~m}$ & $0-1-1$ & $0 \%$ & + & 1419 & $+(7)$ & - & + & - & - & - \\
\hline
\end{tabular}

* Putative DSA (donor typing not available).

Histological evidence suggestive of antibody-mediated rejection was seen in 7 patients; in 5 of these patients this was confirmed by detection of DSA. The following risk factors for BPAR were identified in multivariate regression analyses: BK viral infection, use of cyclosporine as initial CNI, and (with borderline significance) warm ischemic time (data not shown). DGF was not associated with higher rejection rates. Whereas 50\% (16 out of 36) of patients initially treated with cyclosporine developed BPAR, only $28 \%$ (15 out of 53 ) patients on tacrolimus had an acute rejection episode $\left(\chi^{2} p=0.032\right)$.

\section{Preimplantation transplant biopsies}

In 83 (93\%) patients, a preimplantation biopsy could be evaluated. Frequencies of tubular atrophy (absent, mild, moderate, or severe) were $31 \%, 64 \%, 4 \%$, and $1 \%$, respectively. Rates were $43 \%, 51 \%, 5 \%$, and $1 \%$ for different degrees of interstitial fibrosis; $36 \%, 40 \%, 19 \%$, and $5 \%$ for different degrees of glomerulosclerosis; and 24\%, 39\%, 32\%, and 5\% for different degrees of arterio-arteriolar hyalinosis, respectively. The overall mean histological score was $3.5 \pm 2.2 ; 28$ patients (33.7\%) had 


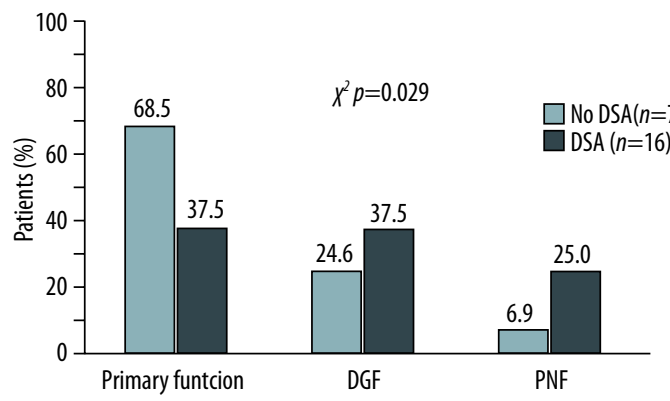

Figure 3. Role of initial graft function and development of DSA. DGF - delayed graft function, PNF - permanent nonfunction or early graft loss.

a total score of less than 3,43 patients $(51.8 \%)$ had a score of 3-5, and 12 patients (14.5\%) had a score >5. Donor creatinine prior to organ procurement and recipient creatinine at 1 year after transplantation were significantly elevated in allografts with a preimplantation biopsy score greater than 5 (Figure 5A). In addition, the percentage of patients with permanent non-function was significantly higher in recipients who received an allograft with a higher histological baseline score (score less than 3: $10.7 \%$, score 3-5: $2.4 \%$, score $>5: 25.0 \%$, $\mathrm{p}=0.034$, Figure $5 \mathrm{~B})$. In multivariate analyses, preimplantation biopsy score was not a predictor of graft outcome in terms of eGFR after 1 year.

\section{Discussion}

The present study was conducted to contribute to a better understanding of the natural course and risk factors of patients 65 years or older who received a kidney transplant from donors of the same age group. In particular, we wanted to extend previous analyses [11-16] by including outcome data that have so far not been studied in detail, including the risk of de-novo DSA development, BK viral infection rate, and tumor development.

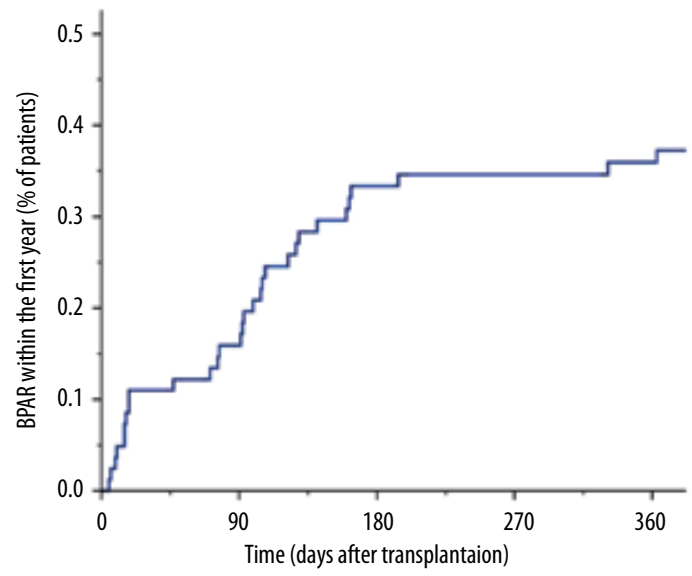

Figure 4. Patients with BPAR episodes within the first year after transplantation.

Our data clearly indicate that ESP patients are at high risk of adverse effects of immunosuppression, such as perioperative surgical complications, susceptibility to infections and sepsis related death, cardiovascular and metabolic morbidity and mortality, and tumor development. On the other hand, ESP recipients also are a group of patients at high risk for BPAR episodes within the first year after transplantation. These findings underscore the dilemma of balancing tailored immunosuppression with control of adverse effects to optimize graft and patient survival in this critical group of patients.

Our findings regarding graft and patient survival are in line with similar-sized studies published in the literature. In these studies, 1- and 5-year allograft survival rates in surviving patients ranged between $85-90 \%$ and $65-80 \%$, while patient survival rates were slightly lower at $80-90 \%$ and $60-75 \%$, respectively [6,10-15].

With respect to patient death, 3 major causes were identified: sepsis, cardiovascular events, and malignancies. These
A

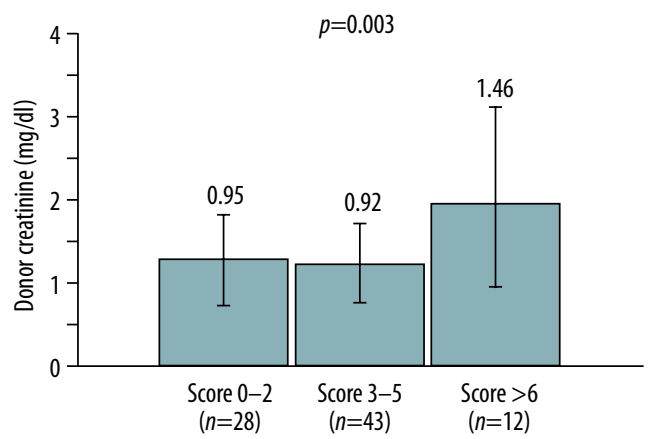

B

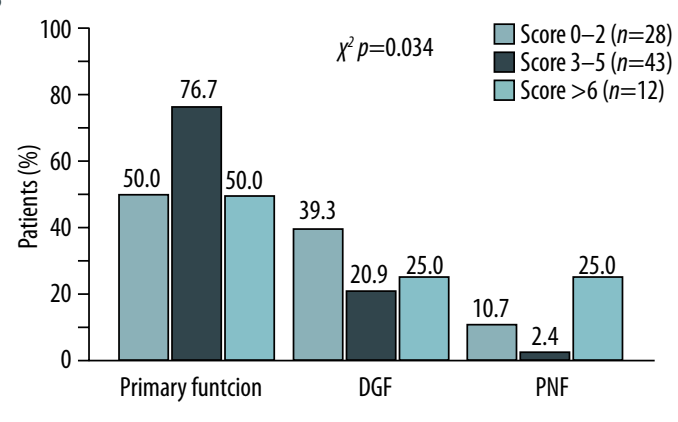

Figure 5. (A) Preimplantation biopsy score and donor creatinine at organ procurement. (B) Preimplantation biopsy score and initial allograft function. 
findings highlight the need for careful selection criteria and critical assessment of patient status, with special attention to comorbidities such as underlying cardiovascular or unknown occult tumor disease. Furthermore, vulnerability to sepsis emphasizes the need to optimize immunosuppression together with close surveillance of patients. Since older individuals with comorbidities are more prone to infectious diseases, implementation of less-potent immunosuppressive regimens could be advocated. On the other hand, BPAR rates in our study cohort were quite high, but still compatible with reports by others $[11,13,15]$. However, graft loss due to chronic rejection was a rare event. In our cohort, BPAR was more frequent in patients treated with cyclosporine as initial CNI. If not predetermined by study inclusion criteria, the choice of initial CNI at our institution is largely based on the underlying immunological risk and the vascular and metabolic profile of the patient. Notably, risk of infection (including BK-viremia), risk of tumor disease, and, above all, patient survival were not affected by choice of initial CNI or induction therapy (ATG vs. basiliximab). Owing to the increased risk of infection and the high rate of BPAR, which mostly occurred within the first 6 months after transplantation, we recently updated our institutional guidelines by recommending a protocol biopsy 3 months after transplantation in ESP patients. Furthermore, since 2013 we recommend the use of ATG as induction therapy in combination with cyclosporine to achieve early reduction of $\mathrm{CNI}$ trough levels. The rationale for tailored immunosuppression is to control adverse effects while still providing sufficiently potent immunosuppression in the early phase after transplantation in order to avoid BPAR. This concept is also based on recent evidence suggesting a potent early immune response in ESP transplant recipients $[16,17]$. These individuals showed stronger T-cell alloreactivity at all time-points observed [16]. In line with these findings, we observed a greater likelihood of DSA development in patients with DGF or permanent non-function.

In agreement with Giessing et al., we did not find a negative impact of prolonged cold ischemia time in successively transplanted kidneys from the same donor [18]. On the other hand, results from the largest ESP study published so far indicate that each hour of prolonged cold ischemia increases the probability of graft loss by $3 \%$ [6]. Although we were not able to document a difference in our cohort, we suggest that kidneys coming from the same donor should be transplanted in parallel whenever this is logistically feasible.

The observed peri-postoperative complication rate of $\sim 40 \%$ observed in our study cohort (most of which were surgeryrelated) is also compatible with recent observations by others $[10,11]$. For example, Bentas et al. reported a surgical complication rate of $47 \%[10]$ and Bodingbauer described a similar surgical complication rate of $41 \%$ [11].
The risk of lymphocele formation observed in our study cohort is in line with other studies that have reported frequencies between $15 \%$ and $25 \%[10,11,19]$. The most important risk factors for lymphocele formation reported in the literature are type of immunosuppression and obesity [20]. In a retrospective study, a BMI of $>25 \mathrm{~kg} / \mathrm{m}^{2}$ was identified as a prognostic factor for allograft survival in ESP patients [21]. Our transplant center advocates a BMI of $<30 \mathrm{~kg} / \mathrm{m}^{2}$ in all patients on the waiting list, but $18 \%$ of our patients missed this goal. These patients should be informed about the greater risk of wound complications and other adverse effects such as development of NODAT following transplantation.

In our study, $17 \%$ of all patients had post-transplant malignancies, most of which were skin tumors. This is in line with an overall tumor incidence rate of $13.7 \%$ in 1882 kidney recipients transplanted in our center between 1966 and 2005 [22]. In a recent analysis of the United Network for Organ Sharing (UNOS), the occurrence of post-transplant malignancies was evaluated in 123380 kidney transplant recipients who received their allografts between 1999 and 2008 [23]. In this younger cohort, $3.39 \%$ of all patients developed a non-skin tumor [23]. Excluding skin tumors, we still find a rate of tumor development of $\sim 9 \%$ in ESP patients. However, this is not surprising, since age was strongly associated with tumor disease in the aforementioned study [23]. Accordingly, patients in the age category 60-69 years had a 5-fold higher risk for tumor development compared to those aged 20-29 years [23]. Overall, kidney transplant recipients are at greatest risk for skin tumors. A cohort of 7952 kidney transplant patients from Sweden who were transplanted between 1970 and 2008 showed a 50-fold higher risk for squamous cell carcinoma within the first 4 years after transplantation, which rose to 160 -fold after $10-19$ years [24]. These findings underscore the importance of regular screening for skin tumors in transplant recipients and of special attention to targeted immunosuppression and UV light protection.

The value of zero-hour biopsies in transplantation of kidneys from older donors was first emphasized by Remuzzi et al. [25]. In a cohort of 62 patients with either single or dual kidney transplantation, preimplantation biopsy and donor age were the only significant predictors for allograft survival [25]. Irrespective of donor creatinine clearance prior to organ procurement, longterm allograft survival of kidneys with a preimplantation biopsy was $21 \%$ better than that of single grafts allocated without biopsies [25]. At our institution the results of preimplantation biopsies are not available prior to transplantation, mainly due to logistic reasons. Organ procurement, subsequent allocation, and transplantation usually occur during the night or in the early morning hours, at which time we cannot provide service for histological evaluation of biopsy samples. Furthermore, histological analysis of frozen sections is often very limited. In addition, preimplantation biopsies are done during back-table 
preparation and processing, and histological evaluation would delay transplantation of these organs. In our study, preimplantation biopsy score did predict initial graft function but was not associated with eGFR at later time-points.

In the present study we also analyzed the development of HLA alloantibodies and DSA, which are increasingly recognized as a major risk factor for kidney transplant failure and are associated with $40 \%$ lower graft survival after 10 years [26,27]. The frequency of de-novo DSA observed in our study is within the $15-25 \%$ range reported in kidney transplantation $[28,29]$. The most common DSA detected are directed against HLA class II antigens, especially against HLA-DQ [28,29]. We confirmed these findings in our study. Since ESP kidneys are not as well HLA-matched, this issue is critical and strategies for class II epitope matching are being developed.

Our study is limited due to its retrospective nature and the rather small sample size. However, the latter allowed us to meticulously analyze a variety of different outcome parameters with no loss during follow-up. A further limitation of our study is the lack of detailed information of donor characteristics, which may influence subsequent allograft survival. Accordingly, Iwan-Zietek et al. reported an enhanced risk of perioperative complications in transplant recipients who received a renal allograft from aged kidney donors who died from cerebral ischemia or bleeding [30]. In these complicated kidney donors they observed increased activity of blood coagulation, which may explain early graft losses due to thrombosis.

\section{References:}

1. Cecka JM, Cohen B, Rosendale J et al: Could more effective use of kidneys recovered from older deceased donors result in more kidney transplants for older patients? Transplantation, 2006; 81(7): 966-70

2. Wolfe RA, Ashby VB, Milford EL et al: Comparison of mortality in all patients on dialysis, patients on dialysis awaiting transplantation, and recipients of a first cadaveric transplant. N Engl J Med, 1999; 341(23): 1725-30

3. Cohen B, Smits JM, Haase B et al: Expanding the donor pool to increase renal transplantation. Nephrol Dial Transplant, 2005; 20(1): 34-41

4. Schaubel D, Desmeules M, Mao Y et al: Survival experience among elderly end-stage renal disease patients. A controlled comparison of transplantation and dialysis. Transplantation, 1995; 60(12): 1389-94

5. Smits JM, Persijn GG, van Houwelingen $\mathrm{HC}$ et al: Evaluation of the Eurotransplant Senior Program. The results of the first year. Am J Transplant, 2002; 2(7): 664-70

6. Frei U, Noeldeke J, Machold-Fabrizii V et al: Prospective age-matching in elderly kidney transplant recipients - a 5-year analysis of the Eurotransplant Senior Program. Am J Transplant, 2008; 8(1): 50-57

7. Matas AJ, Smith JM, Skeans MA et al: OPTN/SRTR 2012 Annual Data Report: kidney. Am J Transplant, 2014; 14(Suppl.1): 11-44

8. Jacobi J, Rebhan D, Heller $\mathrm{K}$ et al: Donor acute kidney injury and shortterm graft outcome in renal transplantation. Clin Transplant, 2014; 28(10): 1131-41

9. Masson I, Maillard N, Cavalier E et al: KDIGO guidelines and kidney transplantation: Is the cystatin-c based recommendation relevant? Am J Transplant, 2015; 15(8): 2211-14

10. Bentas W, Jones J, Karaoguz A et al: Renal transplantation in the elderly: surgical complications and outcome with special emphasis on the Eurotransplant Senior Programme. Nephrol Dial Transplant, 2008; 23(6): 2043-51

\section{Conclusions}

Our data confirm that patients transplanted within the ESP program should be considered as a high-risk patient population; they require close surveillance and, in particular, special attention to balance immunosuppression and prevent cardiovascular and infectious complications. Keeping in mind the devastating prognosis of aged dialysis patients, we believe that elderly transplant recipients still benefit from transplantation if carefully evaluated and selected prior to transplantation and closely monitored and followed thereafter.

\section{Acknowledgments}

We are indebted to Antje Weixelbaum and Dr. Sabine Jank for recruiting patients for follow-up visits.

\section{Conflict of interests}

Since 2012, Novartis provides financial support for the maintenance and data entry of the transplant database, but Novartis had no impact on data acquisition and interpretation.

The results presented in this paper have not been published previously in whole or in part.

11. Bodingbauer M, Pakrah B, Steininger R et al: The advantage of allocating kidneys from old cadaveric donors to old recipients: a single-center experience. Clin Transplant, 2006; 20(4): 471-75

12. Chavalitdhamrong D, Gill J, Takemoto $S$ et al: Patient and graft outcomes from deceased kidney donors age 70 years and older: an analysis of the Organ Procurement Transplant Network/United Network of Organ Sharing database. Transplantation, 2008; 85(11): 1573-79

13. Fabrizii $\mathrm{V}$, Kovarik J, Bodingbauer $M$ et al: Long-term patient and graft survival in the eurotransplant senior program: a single-center experience. Transplantation, 2005; 80(5): 582-89

14. Foss A, Heldal $\mathrm{K}$, Scott $\mathrm{H}$ et al: Kidneys from deceased donors more than 75 years perform acceptably after transplantation. Transplantation, 2009; 87(10): 1437-41

15. Giessing $M$, Conrad S, Schonberger B et al: [Kidney donors and kidney transplantation in the elderly]. Urologe A, 2004; 43(8): 947-54 [in German]

16. Pratschke J, Merk V, Reutzel-Selke $A$ et al: Potent early immune response after kidney transplantation in patients of the European senior transplant program. Transplantation, 2009; 87(7): 992-1000

17. de Fijter JW, Mallat MJ, Doxiadis II et al: Increased immunogenicity and cause of graft loss of old donor kidneys. J Am Soc Nephrol, 2001; 12(7): 1538-46

18. Giessing M, Fuller TF, Friedersdorff F et al: Comparison of first and second kidney transplants from the same deceased donor. Nephrol Dial Transplant, 2010; 25(12): 4055-61

19. Hernandez D, Rufino $M$, Armas $S$ et al: Retrospective analysis of surgical complications following cadaveric kidney transplantation in the modern transplant era. Nephrol Dial Transplant, 2006; 21(10): 2908-15 
20. Mehrabi A, Fonouni $H$, Wente $M$ et al: Wound complications following kidney and liver transplantation. Clin Transplant, 2006; 20(Suppl.17): 97-110

21. Bahde R, Vowinkel T, Unser J et al: Prognostic factors for kidney allograft survival in the Eurotransplant Senior Program. Ann Transplant, 2014; 19: 201-9

22. Apel H, Walschburger-Zorn $\mathrm{K}$, Haberle $\mathrm{L}$ et al: De novo malignancies in renal transplant recipients: experience at a single center with 1882 transplant patients over 39 yr. Clin Transplant, 2013; 27(1): E30-36

23. Sampaio MS, Cho YW, Qazi Y et al: Posttransplant malignancies in solid organ adult recipients: an analysis of the U.S. National Transplant Database. Transplantation, 2012; 94(10): 990-98

24. Krynitz B, Edgren G, Lindelof B et al: Risk of skin cancer and other malig nancies in kidney, liver, heart and lung transplant recipients 1970 to 2008 - a Swedish population-based study. Int J Cancer, 2013; 132(6): 1429-38
25. Remuzzi G, Cravedi P, Perna A et al: Long-term outcome of renal transplantation from older donors. N Engl J Med, 2006; 354(4): 343-52

26. Mao Q, Terasaki PI, Cai J et al: Extremely high association between appearance of HLA antibodies and failure of kidney grafts in a five-year longitudinal study. Am J Transplant, 2007; 7(4): 864-71

27. Worthington JE, Martin S, Al-Husseini DM et al: Posttransplantation production of donor HLA-specific antibodies as a predictor of renal transplant outcome. Transplantation, 2003; 75(7): 1034-40

28. Kaneku H: 2012 annual literature review of donor-specific HLA antibodies after organ transplantation. Clin Transpl, 2012: 207-17

29. Wiebe C, Pochinco D, Blydt-Hansen TD et al: Class II HLA epitope matching-A strategy to minimize de novo donor-specific antibody development and improve outcomes. Am J Transplant, 2013; 13(12): 3114-22

30. Iwan-Zietek I, Zietek Z, Sulikowski T et al: Impact of kidney donor hemostasis on risk of complications after transplantation - preliminary outcomes. Med Sci Monit, 2013; 19: 1102-8 